Young-Shik Kim

Jang-Pyo Hong

Yun-Ho Kim $\square$

Hong-Gun Sung

Jinwhan Kim

https://doi.org/10.21278/TOF.453029021

ISSN 1333-1124

eISSN 1849-1391

\title{
DESIGN AND EXPERIMENTAL VALIDATION OF A CONTROL SYSTEM FOR DYNAMIC POSITIONING OF A SHUTTLE TANKER
}

\begin{abstract}
Summary
In this paper, a dynamic positioning performance evaluation procedure for a shuttle tanker is discussed through experimental and numerical analyses. A dynamically positioned shuttle tanker with six thrusters (three tunnel thrusters, two azimuth thrusters, and one main propeller with a rudder), operated in deep water condition was considered. A conventional proportional-derivative control algorithm was adopted for the main feedback control algorithm to reduce the position error, and an anti-windup integral control algorithm was introduced to suppress the steady-state error in the dynamic positioning operation. A minimum power consumption algorithm, based on the Lagrange multiplier method, was utilised in the thrust allocation for the thruster systems. An extended Kalman filter was used in the experiment to separate the low-frequency motion from the measured vessel motion. A set of experiments and numerical analyses were conducted in this study under the same environmental conditions and with the same control methodology. The dynamic positioning operation results obtained by the experiments and numerical simulations were compared to evaluate the station-keeping performance of the dynamically positioned shuttle tanker.
\end{abstract}

Key words: $\quad$ Dynamic positioning, shuttle tanker, anti-windup integral control, model test, simulation, deep water condition

\section{Introduction}

The offshore industry faces challenges of moving toward deeper waters in order to find more abundant reservoirs. Moreover, marine operations in the offshore industry are getting more complicated to achieve better operability. Under these circumstances, dynamic positioning (DP) techniques are becoming more common and necessary in the offshore industry. A moored floating production storage and offloading (FPSO) unit offloads oil and gas to a DP shuttle tanker, which then transfers them to the primary storage near the consumer. A supply vessel that is normally a shuttle tanker uses dynamic positioning techniques to follow the moving target vessel, i.e. an FPSO. The FPSO switches from a mooring configuration mode with a submerged turret loading (STL) system to a dynamic positioning mode in case of heavy environmental conditions. These are typical examples of 
the usage of DP techniques [1]. To perform the offloading of oil and gas from an FPSO to a shuttle tanker, it is important to have enough stand-alone station-keeping performance using the DP system for any vessel. In this study, a systematic and comprehensive performance evaluation procedure of a DP system for a shuttle tanker is presented.

Experiments on DP vessels are commonly combined with simulations in order to evaluate the DP performance of the vessels in irregular waves, current, and wind conditions. The evaluation of DP performance is an element of the design procedure for DP vessels. In the first stage of designing a DP vessel, a static capability examination of the DP system is performed under pre-specified environmental conditions to decide on the capacity of the thruster systems. Then, model testing and time-domain simulations are carried out with the decided thruster systems to assess the dynamic performance of the DP vessel. Through this procedure, the DP vessel is designed, and the performance of the DP system is evaluated [2].

In general, a DP control system consists of a low-frequency (LF) motion estimator, a controller, and a thrust allocator. The LF motion estimator separates LF motion from the measured motion of a vessel. When a vessel experiences external ocean forces induced by wind, current, and waves, the vessel is influenced by the wave frequency motion, the slow varying motion, and the mean drift motion. The DP system attempts to reduce the slow varying motion and the mean drift motion components; these two combined motions are called the LF motion of the vessel. It is known that the control of the high-frequency (HF) motion (or wave frequency motion) requires an enormous amount of power and causes extra wear and tear in the mechanical system of the thrusters, which is not a practical solution at all. Thus, the DP control system does not need to treat the HF motion. Therefore, the LF motion estimator is required in the DP control system. In this study, an extended Kalman filter (EKF) was implemented in the DP control system for the model test.

A proportional-derivative (PD) control loop with a wind feed-forward control algorithm is commonly used in DP control systems. Such a wind feed-forward control is achieved using wind sensor measurements; it can compensate for steady wind force effects which would otherwise cause a steady-state error in DP operation [3-5]. Current force effects are generally not considered explicitly in the feed-forward control loop because measurements of current cannot be obtained within a sufficient margin of error by using existing current sensors. Since the current and the waves are strongly coupled near a floating vessel, it is difficult to measure the current independently. Nowadays, techniques for estimating LF force components (which are caused by the wind, current, and 2nd order wave forces) from the measured vessel motion can sometimes be used [6, 7]. An anti-windup integral (I) control algorithm was introduced in this study to avoid the steady-state mean drift error which is caused by the wind, current, and 2nd order wave forces [8].

A DP vessel usually has a larger number of thrusters than the degrees-of-freedom (DOFs) of the vessel motion. This overdetermined characteristic of the DP vessel means that there is more than one solution to the thrust allocation problem. Thus, the thrust allocation problem is considered as an optimization problem under several constraints. In this study, a minimum power consumption algorithm, based on the Lagrange multiplier method, was implemented for the thrust allocation problem.

The aim of this study is to present a systematic and comprehensive performance evaluation procedure of a DP system for a shuttle tanker, which includes control system design, numerical simulations, and model experiments. The remainder of this paper is organized as follows. The equations of motion for a DP vessel are briefly summarized in the next section. The DP control algorithm and thrust allocation algorithm used in this study are introduced. The model test setup and the framework for the numerical simulation are also given. The results obtained by the model testing and numerical simulation are discussed. Furthermore, the station-keeping performance of the DP shuttle tanker is discussed with 
reference to the experimental and numerical simulation results. A series of model tests was conducted in the Ocean Engineering Basin (OEB) of Korea Research Institute of Ships and Ocean Engineering (KRISO).

\section{Equations of motion for a DP vessel}

Low-frequency equations of motion for a floating vessel are generally used in the design and simulation of DP vessels. The DP operation is a function of a vessel which makes the vessel keep its position in a designated, specific area, and overcome environmental forces by using the action of the thruster system. Thus, only horizontal motions in three degrees of freedom (3 DOF; the surge, sway, and yaw motions) were considered in the DP analysis in the deep water condition.
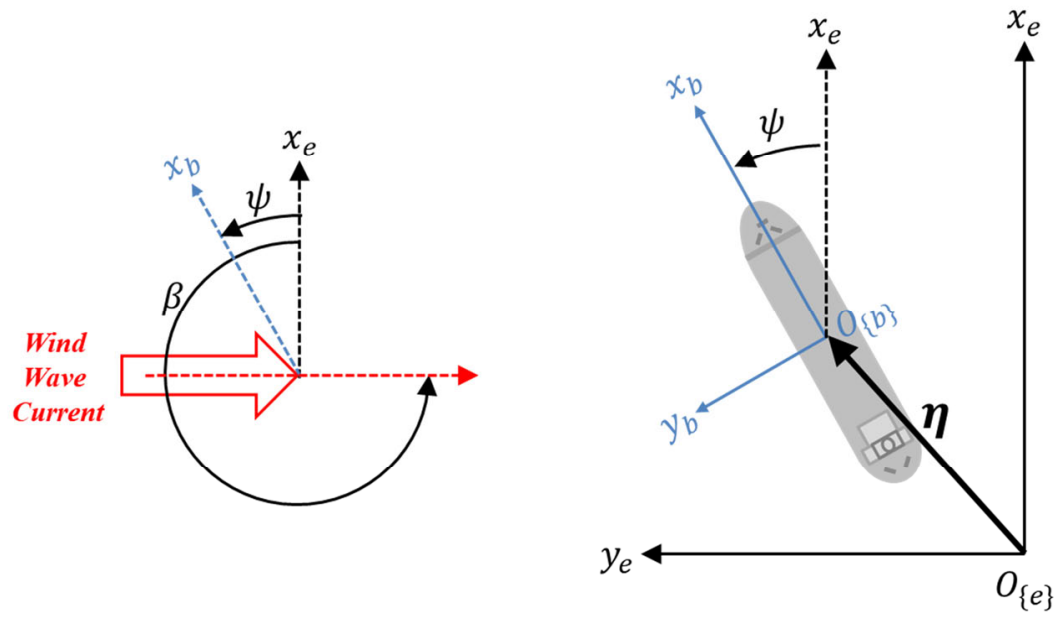

Fig. 1 Coordinate system for the dynamic positioning vessel: the earth-fixed frame $\{e\}$, the body-fixed frame $\{b\}$, and definition of the environmental force directions

In this paper, a mathematical model of a shuttle tanker was considered for DP operation. An earth-fixed frame $\{e\}$ and a body-fixed frame $\{b\}$ were defined, as shown in Figure 1, to establish the low-frequency equations of motion of a vessel. It has been assumed that the origin of the body-fixed frame coincides with the centre of gravity of the vessel while its axes are along the principal axes of inertia of the vessel. Hence, the kinematic equation of a vessel on the horizontal plane can be written as equation (1). Here, $\boldsymbol{\eta}$ is a column vector representing the position of the vessel in $\{e\}$ and the elements $x, y, \psi$ represent the position and the heading angle in the $x_{e}$-axis and $y_{e}$-axis and about the $z_{e}$-axis. The vector $\boldsymbol{v}$ is also a column vector, meaning $3 \mathrm{DOF}$ velocities in $\{b\}$, and the elements $u, v, r$ represent the velocity in the surge, sway, and yaw directions, respectively. The variable $\boldsymbol{J}$ is a Jacobian matrix defining the transform from the motion in $\{b\}$ to the motion in $\{e\}$. Finally, $\dot{\boldsymbol{\eta}}$ is the time derivative of $\boldsymbol{\eta}$.

$\dot{\eta}=J v$

$$
\text { where, } \quad \begin{aligned}
\boldsymbol{\eta} & =\left[\begin{array}{lll}
x & y & \psi
\end{array}\right]^{T}, \\
\boldsymbol{v} & =\left[\begin{array}{lll}
u & v & r
\end{array}\right]^{T}, \\
\boldsymbol{J} & =\left[\begin{array}{ccc}
\cos \psi & -\sin \psi & 0 \\
\sin \psi & \cos \psi & 0 \\
0 & 0 & 1
\end{array}\right] .
\end{aligned}
$$

It is assumed that the velocity of the DP vessel is near zero. This assumption ensures that the added mass and damping at zero frequency affect the whole DP operation. Hence, the dynamic equation of the DP vessel can be formulated as follows: 


$$
\left(M_{R B}+\boldsymbol{a}(0)\right) \dot{\boldsymbol{v}}+\boldsymbol{C}_{R B}(\boldsymbol{v}) \boldsymbol{v}+\boldsymbol{C}_{a}\left(\boldsymbol{v}_{r}\right) \boldsymbol{v}_{r}+\boldsymbol{D}(0) \boldsymbol{v}+\boldsymbol{d N L}\left(\boldsymbol{v}_{r}\right)=\boldsymbol{\tau}_{\text {wind }}+\boldsymbol{\tau}_{\text {wave }}+\boldsymbol{\tau} .
$$

Assuming the longitudinal $x_{b} z_{b}$-plane symmetry in $\{b\}$, the individual components in the dynamic model can be written as follows:

$\boldsymbol{M}_{R B}+\boldsymbol{a}(0)=\left[\begin{array}{ccc}m & 0 & 0 \\ 0 & m & m x_{g}^{b} \\ 0 & m x_{g}^{b} & I_{z}\end{array}\right]+\left[\begin{array}{ccc}-X_{\dot{u}} & 0 & 0 \\ 0 & -Y_{\dot{v}} & -Y_{\dot{r}} \\ 0 & -Y_{\dot{r}} & -N_{\dot{r}}\end{array}\right]$,

$\boldsymbol{C}_{R B}(\boldsymbol{v})=\left[\begin{array}{ccc}0 & 0 & -\left(x_{g}^{b} r+v\right) \\ 0 & 0 & m u \\ \left(x_{g}^{b} r+v\right) & -m u & 0\end{array}\right]$,

$\boldsymbol{C}_{a}\left(\boldsymbol{v}_{r}\right)=\left[\begin{array}{ccc}0 & 0 & Y_{\dot{v}} v_{r}+Y_{\dot{r}} r \\ 0 & 0 & -X_{\dot{u}} u_{r} \\ -\left(Y_{\dot{v}} v_{r}+Y_{\dot{r}} r\right) & X_{\dot{u}} u_{r} & 0\end{array}\right]$,

where, $\quad \boldsymbol{v}_{r}=\left[\begin{array}{c}u_{r} \\ v_{r} \\ r\end{array}\right]=\left[\begin{array}{c}u-u_{c} \\ v-v_{c} \\ r\end{array}\right]=\left[\begin{array}{c}u-V_{c} \cos \left(\beta_{c}-\psi\right) \\ v-V_{c} \sin \left(\beta_{c}-\psi\right) \\ r\end{array}\right]$,

$\boldsymbol{D}(0)=\left[\begin{array}{ccc}-X_{u} & 0 & 0 \\ 0 & -Y_{v} & -Y_{r} \\ 0 & -N_{v} & -N_{r}\end{array}\right]$

$\boldsymbol{d} \boldsymbol{N} \boldsymbol{L}\left(\boldsymbol{v}_{r}\right)=-\frac{1}{2} \rho_{w} V_{r c}{ }^{2}\left[\begin{array}{c}B T C_{x}\left(\gamma_{c}\right) \\ L T C_{y}\left(\gamma_{c}\right) \\ L^{2} T C_{x y}\left(\gamma_{c}\right)\end{array}\right]+\left[\begin{array}{c}0 \\ d_{2} \\ d_{6}\end{array}\right]$

In equation (3), $\boldsymbol{M}_{R B}$ is the rigid body mass matrix expressed in $\{b\}, m$ is the rigid body mass, $x_{g}^{b}$ is the position of the center of gravity (COG) expressed in $\{b\}$, and $I_{z}$ is the rigid body inertia with respect to the $z$ axis of the body-fixed frame $\{b\}$. It should be noticed that the body-fixed frame has not been positioned in the COG. Then $\boldsymbol{a}(0)$ is the added mass matrix at the zero frequency which is calculated from the 3D potential code of the Korea Research Institute of Ships and Ocean Engineering (KRISO) [9]. The variables $X_{\dot{u}}, Y_{\dot{v}}, Y_{\dot{r}}, N_{\dot{r}}$ are coefficients for the surge added mass due to surge acceleration, sway added mass due to sway acceleration, sway added mass due to yaw acceleration, and yaw added mass due to yaw acceleration, respectively. This added mass coefficient expression is in accordance with the notation of the Society of Naval Architects and Marine Engineers (SNAME) [10]. The term $\boldsymbol{C}_{R B}(\boldsymbol{v})$ in equation (4) is the coefficient matrix of the Coriolis and the centrifugal force caused by the rigid body motion. In equation (5), $\boldsymbol{C}_{a}\left(\boldsymbol{v}_{r}\right)$ is the Coriolis and centrifugal force coefficient matrix due to the added mass, which generates the nonlinear Munk moment by multiplication with the relative velocities of the vessel. The Munk moment is known to have destabilizing effects on the yaw motion of the vessel. The vessel velocity relative to the current flow is expressed as $\boldsymbol{v}_{r}$ while the variables $u_{c}$ and $v_{c}$ are the ocean current velocity components expressed in $\{b\}$. The current speed and the direction in $\{e\}$ are defined as $V_{c}$ and $\beta_{c}$, respectively. In equation $(6), \boldsymbol{D}(0)$ is the linear damping coefficient matrix. The coefficients in the matrix are in accordance with the notation of the SNAME; $X_{u}, Y_{v}, Y_{r}, N_{v}$, $N_{r}$ are respectively the surge damping coefficient due to surge velocity, sway damping coefficient due to sway velocity, sway damping coefficient due to yaw velocity, yaw damping coefficient due to sway velocity, and yaw damping coefficient due to yaw velocity. In 
equation (7), $\boldsymbol{d} \boldsymbol{N} \boldsymbol{L}\left(\boldsymbol{v}_{r}\right)$ is the nonlinear damping force vector calculated by the Oil Companies International Marine Forum (OCIMF) method and the cross-flow drag theory [11-14]. Here, $\rho_{w}$ is the density of water, $V_{r c}$ is the relative speed of the current, and $L, B, T$ are the length, breadth, and draft of the vessel, respectively. The terms $C_{x}\left(\gamma_{c}\right), C_{y}\left(\gamma_{c}\right), C_{x y}\left(\gamma_{c}\right)$ are the current force and moment coefficients for the surge, sway, and yaw directions with the angle of attack of the current $\gamma_{c}$. Note that the current force coefficients should be estimated before applying nonlinear current damping; this can be done through the basin model test, the wind tunnel test, or proper estimation, such as interpolation with the length and width of a vessel based on the OCIMF data and calculation by computational fluid dynamics. Here, the relative speed of the current $V_{r c}$ and the angle of attack of the current $\gamma_{c}$ are defined as follows:

$$
\begin{aligned}
& V_{r c}=\sqrt{u_{r}^{2}+v_{r}^{2}}=\sqrt{\left(u-u_{c}\right)^{2}+\left(v-v_{c}\right)^{2}}, \\
& \gamma_{c}=\tan ^{-1}\left(\frac{v_{r}}{u_{r}}\right)=\tan ^{-1}\left(\frac{v-v_{c}}{u-u_{c}}\right) .
\end{aligned}
$$

The nonlinear damping forces caused by the cross-flow along the vessel hull are given as $d_{2}$ and $d_{6}$. Here, $d_{2}$ and $d_{6}$ are the cross-flow damping force and moment in the sway and yaw directions, respectively, and the formulations are given as follows:

$$
\begin{aligned}
& d_{2}=\frac{1}{2} \rho_{w} T C_{y}\left(\frac{\pi}{2}\right) \int_{-L / 2}^{L / 2}\left[\left(v_{r}-r x\right)\left|v_{r}-r x\right|-v_{r}\left|v_{r}\right|\right] \mathrm{d} x, \\
& d_{6}=\frac{1}{2} \rho_{w} T C_{y}\left(\frac{\pi}{2}\right) \int_{-L / 2}^{L / 2} x\left[\left(v_{r}-r x\right)\left|v_{r}-r x\right|-v_{r}\left|v_{r}\right|\right] \mathrm{d} x .
\end{aligned}
$$

In these equations, $C_{y}(\pi / 2)$ is the current force coefficient, i.e. drag coefficient, in the sway direction at a $90^{\circ}$ incident flow angle.

The wind force $\boldsymbol{\tau}_{\text {wind }}$ has highly nonlinear characteristics. Generally speaking, the OCIMF method is used for wind force generation as shown in equation (12).

$$
\boldsymbol{\tau}_{\text {wind }}=-\frac{1}{2} \rho_{a} V_{r w}{ }^{2}\left[\begin{array}{c}
A_{f} C_{x, w}\left(\gamma_{w}\right) \\
A_{s} C_{y, w}\left(\gamma_{w}\right) \\
A_{s} L C_{x y, w}\left(\gamma_{w}\right)
\end{array}\right],
$$

where, $\quad V_{r w}=\sqrt{\left(u-u_{w}\right)^{2}+\left(v-v_{w}\right)^{2}}$,

$$
\begin{aligned}
& \gamma_{w}=\tan ^{-1}\left(\frac{v-v_{w}}{u-u_{w}}\right), \\
& u_{w}=V_{w} \cos \left(\beta_{w}-\psi\right), \\
& v_{w}=V_{w} \sin \left(\beta_{w}-\psi\right) .
\end{aligned}
$$

Here, $\rho_{a}$ is the air density, $V_{r w}$ is the relative speed of the current, and $A_{f}, A_{s}$ are the projection areas of the vessel hull above the water line at the front and the side, respectively. The terms $C_{x, w}\left(\gamma_{w}\right), C_{y, w}\left(\gamma_{w}\right), C_{x y, w}\left(\gamma_{w}\right)$ are the wind force and moment coefficients for the surge, sway, and yaw directions with the angle of attack of the wind $\gamma_{w}$. It should be noted that the wind force and moment coefficients can be similarly estimated through the wind tunnel test, or proper estimation, such as interpolation with the length and width of a vessel based on the OCIMF data and calculation by computational fluid dynamics. The variables $u_{w}$ and $v_{w}$ used in expressing $V_{r w}$ and $\gamma_{w}$ are the wind velocity components expressed in $\{b\}$. The variable $V_{w}$ is the wind speed and $\beta_{c}$ is the wind direction expressed in $\{e\}$.

In the simulation, slow-drift $2^{\text {nd }}$ order wave forces are considered. The slow-drift $2^{\text {nd }}$ order wave force is constructed with the quadratic transfer functions (QTFs), difference 
frequencies, and phases [15]. The QTFs are calculated from the KRISO 3D potential flow solver. Most of the hydrodynamic codes generate QTF sets with discrete heading angles. Thus, an interpolation method for QTFs should be considered for use in the simulation code. The frequency differences and phase differences are calculated from a wave generation module. The equation of the slow-drift $2^{\text {nd }}$ order wave force $\boldsymbol{\tau}_{\text {wave }}$ in the deep water condition is formulated as follows:

$$
\tau_{\text {wave }}^{i}=\sum_{j=1}^{N} \sum_{k=1}^{N} A_{j} A_{k}\left[\begin{array}{c}
Q T F_{R}^{i}\left(\omega_{j}, \omega_{k}, \psi_{r}\right) \cos \left(\omega_{d} t+\varepsilon_{d}\right) \\
+Q T F_{I}^{i}\left(\omega_{j}, \omega_{k}, \psi_{r}\right) \sin \left(\omega_{d} t+\varepsilon_{d}\right)
\end{array}\right],
$$

where,

$$
\begin{aligned}
& \omega_{d}=\omega_{j}-\omega_{k}, \\
& \varepsilon_{d}=\varepsilon_{j}-\varepsilon_{k}, \\
& \psi_{r}=\beta_{\text {wave }}-\psi,
\end{aligned}
$$

The term $\tau_{\text {wave }}^{i}$ represents the slow-drift $2^{\text {nd }}$ order wave forces and moment for the $i^{\text {th }}$ motion among surge, sway, and yaw; $A_{j}$ and $A_{k}$ are the wave amplitude components at the $j^{t h}$ frequency and $k^{\text {th }}$ frequency components, respectively. These wave amplitude components are defined in the generation of irregular waves from a given wave spectrum. The term $\operatorname{QTF}_{R}^{i}\left(\omega_{j}, \omega_{k}, \psi_{r}\right)$ is the real part of QTF for the $i^{t h}$ motion and $\operatorname{QTF}_{I}^{i}\left(\omega_{j}, \omega_{k}, \psi_{r}\right)$ is the imaginary part. Note that QTF is a function of the two frequency components $\omega_{j}, \omega_{k}$, and the relative vessel heading $\psi_{r}$ with respect to the wave direction. The frequency components, $\omega_{j}$, $\omega_{k}$, are the wave frequency defined in the generation of irregular waves, like $A_{j}$ and $A_{k}$. The term $\beta_{\text {wave }}$ is the wave direction expressed in $\{e\}$. On the other hand, the vessel heading $\psi$ is not predefined. Therefore, generally, the interpolation of QTF with respect to $\psi_{r}$ is required in the wave generation in the time domain simulation. The variables $\omega_{d}$ and $\varepsilon_{d}$ are the frequency difference and the phase difference between the $j^{\text {th }}$ component and $k^{t h}$ component of frequency and phase. It should be noticed that all the frequencies and phase components are the same frequency and phase components in the generation of wave. According to equation 13 , it can be easily seen that the $2^{\text {nd }}$ wave force is proportional to the square of the wave amplitude.

\section{DP control algorithm}

In the experiment, the extended Kalman filter (EKF) was used for the LF motion estimator. The LF motion of the vessel estimated by the EKF was used as the input to the DP controller. On the other hand, in the numerical simulation, only the $2^{\text {nd }}$ order wave force was considered. Therefore, it should be noted that there was no need to use any filtering technology to get the low-frequency motion of the vessel in the simulations.

PD control algorithms are commonly used in DP control systems. The proportional (P) control action behaves like stiffness, and the derivative (D) control action works like a damping effect in the mooring system of the vessel. Thus, it is reasonable to estimate the PD gains from the expected system responses by using calculations for a mass-spring-damper system in the first step of gain tuning for the DP controller. However, an unaltered integral (I) controller is rarely used in DP control systems because the I controller easily makes the system divergent. This characteristic of the I controller is a result of the integration of fluctuating errors; the I controller response is more rapid than the response of the control system. Thus, in this study, an anti-windup I controller was introduced to suppress steadystate errors. Equation 14 gives the error definition in the DP control. In the case of the DP operation of the shuttle tanker, the bow loading system (BLS), which is generally located in the fore of the vessel, was the control target point for maintaining a designated position in 
$\{e\}$. Equation 15 shows a standard PID control algorithm while equation 16 shows the PD control algorithm with the introduced anti-windup I control algorithm. The proposed antiwindup I control algorithm uses the average of errors in a confined time window, and thus it did not respond to fast varying error components. It is recommended that the size of the confined time window $T_{I}$ of the anti-windup I controller should be set to a larger value than the natural period of the control system response to avoid instability of the system.

$\boldsymbol{e}=\boldsymbol{\eta}_{d}-\boldsymbol{\eta}$,

$\boldsymbol{\tau}=\boldsymbol{K}_{P} \boldsymbol{e}+\boldsymbol{K}_{D} \dot{\boldsymbol{e}}+\boldsymbol{K}_{I} \int_{0}^{t} \boldsymbol{e} \mathrm{d} t$

$\boldsymbol{\tau}=\boldsymbol{K}_{P} \boldsymbol{e}+\boldsymbol{K}_{D} \dot{\boldsymbol{e}}+\boldsymbol{F}_{I}$,

where, $\quad \boldsymbol{F}_{I}=\left\{\begin{array}{cl}0, & \text { if } t=0 \\ \boldsymbol{F}_{I}+\left(\boldsymbol{K}_{I} / T_{I}\right) \int_{t-T_{I}}^{t} \boldsymbol{e} \mathrm{d} t, & \text { if } t=n T_{I}, \quad n=1,2,3, \cdots \\ \boldsymbol{F}_{I} & \text { if } t \neq n T_{I}, \quad n=1,2,3, \cdots\end{array}\right.$

The term $\boldsymbol{e}$ is the position error of the vessel, and $\boldsymbol{\eta}_{d}$ is the target position vector of the BLS; $\boldsymbol{\tau}$ represents the calculated control forces and the moment in $\{b\}$, which is a combination of the counteracting forces and moment needed to maintain the planar position of the vessel under the varying ocean environmental conditions; $\dot{\boldsymbol{e}}$ is the derivate of position error with respect to time; $\boldsymbol{K}_{P}, \boldsymbol{K}_{D}$, and $\boldsymbol{K}_{I}$ are the P, D, and I gains for the PD control algorithm; $\boldsymbol{F}_{I}$ represents I control forces and the moment with the proposed anti-windup I control algorithm; the variable $T_{I}$ is the size of the time window of the error integration for the I control algorithm; the variable $n$ is the number of the time window repeats.

\section{Thrust allocation algorithm}

A minimum power consumption algorithm based on the Lagrange multiplier method is used for thrust allocation in this study. The counteracting forces and moment required to maintain the position of the DP vessel within the predefined area for safe marine operations were calculated from the PD control algorithm with an anti-windup I control algorithm. Most DP vessels are over-actuated, which implies that the vessel has a larger number of actuators than the number of DOFs of the vessel motion. In this over-actuated case, there is more than one solution to matching the required forces and the forces generated by the action of the thrusters. Therefore, the thrust allocation problem is regarded as an optimization problem.

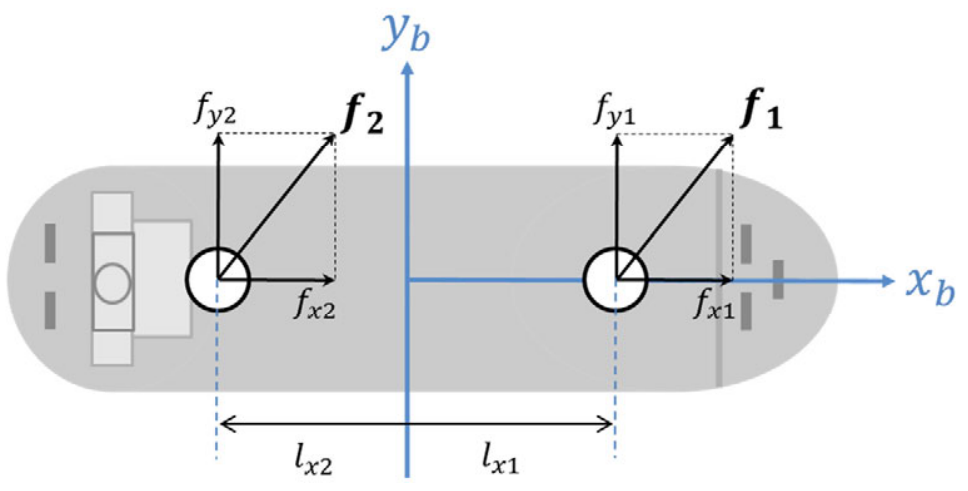

Fig. 2 Example of a simple thruster configuration: two azimuth thrusters

Figure 2 shows a simple thruster configuration with two azimuth thrusters. As stated above, the equilibrium condition between the required forces and moment calculated by the 
controller and the forces and moment generated by the action of the thrusters can be formulated as in equation 17. This equation is called the actuator model.

$$
\boldsymbol{\tau}=\left[\begin{array}{lll}
X & Y & N
\end{array}\right]^{T}=\boldsymbol{T} \boldsymbol{f}=\boldsymbol{T} \boldsymbol{K u},
$$

$$
\text { where, } \quad \begin{aligned}
\boldsymbol{T} & =\left[\begin{array}{cccc}
1 & 0 & 1 & 0 \\
0 & 1 & 0 & 1 \\
0 & l_{x 1} & 0 & -l_{x 2}
\end{array}\right], \\
\boldsymbol{f} & =\left[\begin{array}{llll}
f_{1 x} & f_{1 y} & f_{2 x} & f_{2 y}
\end{array}\right]^{T}, \\
\boldsymbol{K} & =\left[\begin{array}{cccc}
a_{1}{ }^{2} & 0 & 0 & 0 \\
0 & a_{1}{ }^{2} & 0 & 0 \\
0 & 0 & a_{2}{ }^{2} & 0 \\
0 & 0 & 0 & a_{2}{ }^{2}
\end{array}\right], \\
\boldsymbol{u} & =\left[\begin{array}{llll}
u_{1 x} & u_{1 y} & u_{2 x} & u_{2 y}
\end{array}\right]^{T} .
\end{aligned}
$$

The term $\boldsymbol{\tau}$ is the required forces and moment vector expressed in $\{b\}$, the elements $X, Y$, and $N$ are the required forces and moment calculated by the DP controller for the surge, sway, and yaw directions, respectively; $\boldsymbol{T}$ is the thruster configuration matrix and the variables $l_{x 1}, l_{x 2}$ are the distances of the azimuth thruster force vectors $\boldsymbol{f}_{1}, \boldsymbol{f}_{2}$ in the $x_{b}$ direction from the centre of $\{b\}$. In order to consider all kinds of thrusters in the thruster allocation, the generalized thrust that represents thrust in the $x$ - and $y$-components in $\{b\}$ is defined as $\boldsymbol{f}$. Here, the variables $f_{x 1}, f_{x 2}, f_{y 1}$, and $f_{y 2}$ are the force components in the $x_{b}$ and $y_{b}$ directions in $\{b\}$ for the azimuth thruster force vectors $\boldsymbol{f}_{1}$ and $\boldsymbol{f}_{2}$, and $\boldsymbol{K}$ is the thrust coefficient matrix. The variables $a_{1}, a_{2}$ in $\boldsymbol{K}$ are the thrust-revolutions per second (RPS) relationship coefficients of the thruster. Generally, the propeller rotational speed has a quadratic relationship with the thrust generated by the propeller. The thrust-RPS relationship coefficient is generally obtained by the bollard pull test. Finally, $\boldsymbol{u}$ is the control input command for thrusters in the $x_{b}$ and $y_{b}$ directions in $\{b\}$. It should be noted that the propeller rotational speed $\left(u_{1}, u_{2}\right)$ and the azimuth angle $\left(\alpha_{1}, \alpha_{2}\right)$ are calculated from $u_{1 x}, u_{1 y}, u_{2 x}$, and $u_{2 y}$, as shown in equations $18 \mathrm{a}$ and $18 \mathrm{~b}$.

$$
\begin{array}{ll}
u_{1}=\sqrt{\left(u_{\left.1 x^{2}+u_{1 y}^{2}\right)}\right.}, \quad \alpha_{1}=\tan ^{-1}\left(u_{1 y} / u_{1 x}\right), \\
u_{2}=\sqrt{\left(u_{\left.2 x^{2}+u_{2 y}{ }^{2}\right)}\right.}, \quad \alpha_{2}=\tan ^{-1}\left(u_{2 y} / u_{2 x}\right),
\end{array}
$$

From the actuator model, note that the thruster configuration matrix $\boldsymbol{T}$ can be square or non-square. This implies that the number of control inputs is equal to or greater than the number of DOFs of the vessel motion. It is possible to find an optimal distribution of control forces for each direction by using the explicit method. This thrust allocation problem can be considered as an unconstrained least-squares optimization problem, which is written in equation 19.

$J=\min _{\boldsymbol{f}}\left\{\boldsymbol{f}^{T} \boldsymbol{W} \boldsymbol{f}\right\} \quad$ subject to: $\boldsymbol{T} \boldsymbol{f}-\boldsymbol{\tau}=\mathbf{0}$,

where $\boldsymbol{W}$ is a diagonal positive definite weighting matrix. Normally, the weighting matrix $\boldsymbol{W}$ has an inverse relationship with the maximum thrust for each thruster. Thus, the weighting effect can distribute the required thrust within the capability of each thruster. 
To solve the optimization problem, let's consider the Lagrange function for the cost function formulation.

$L(\boldsymbol{f}, \boldsymbol{\lambda})=\boldsymbol{f}^{T} \boldsymbol{W} \boldsymbol{f}+\boldsymbol{\lambda}^{T}(\boldsymbol{T} \boldsymbol{f}-\boldsymbol{\tau})$,

where $\lambda$ is a vector of Lagrange multipliers.

To find the extreme value of the Lagrange function, set

$\frac{\partial L(\boldsymbol{f}, \lambda)}{\partial\left[\begin{array}{ll}\boldsymbol{f}^{T} & \lambda^{T}\end{array}\right]^{T}}=\left[\begin{array}{c}2 \boldsymbol{W} \boldsymbol{f}-\boldsymbol{T}^{T} \boldsymbol{\lambda} \\ \boldsymbol{\tau}-\boldsymbol{T} \boldsymbol{f}\end{array}\right]=0$.

After rearranging equation 21 with the vectors $\boldsymbol{f}$ and $\boldsymbol{\lambda}$, the linear matrix equation can be obtained as

$$
\left[\begin{array}{cc}
2 \boldsymbol{W} & -\boldsymbol{T}^{T} \\
\boldsymbol{T} & \mathbf{0}
\end{array}\right]\left[\begin{array}{l}
\boldsymbol{f} \\
\lambda
\end{array}\right]=\left[\begin{array}{l}
\mathbf{0} \\
\boldsymbol{\tau}
\end{array}\right]
$$

Hence, the optimal generalized thrust can be calculated with an inverse of the matrix as follows:

$$
\left[\begin{array}{l}
\boldsymbol{f} \\
\lambda
\end{array}\right]=\left[\begin{array}{cc}
2 \boldsymbol{W} & -\boldsymbol{T}^{T} \\
\boldsymbol{T} & \mathbf{0}
\end{array}\right]^{-1}\left[\begin{array}{l}
\mathbf{0} \\
\boldsymbol{\tau}
\end{array}\right]
$$

Considering the relationship of the generalized thrust $\boldsymbol{f}$ and the control input command $\boldsymbol{u}$, equation 23 can be reformed as equation 25 .

$$
\begin{aligned}
& {\left[\begin{array}{l}
\boldsymbol{f} \\
\lambda
\end{array}\right]=\left[\begin{array}{c}
\boldsymbol{K u} \\
\boldsymbol{\lambda}
\end{array}\right]=\left[\begin{array}{cc}
2 \boldsymbol{W} & -\boldsymbol{T}^{T} \\
\boldsymbol{T} & \mathbf{0}
\end{array}\right]^{-1}\left[\begin{array}{l}
\mathbf{0} \\
\boldsymbol{\tau}
\end{array}\right],} \\
& {\left[\begin{array}{l}
\boldsymbol{u} \\
\boldsymbol{\lambda}
\end{array}\right]=\left[\begin{array}{cc}
\boldsymbol{K}^{-1} & \mathbf{0} \\
\mathbf{0} & \boldsymbol{I}
\end{array}\right]\left[\begin{array}{cc}
2 \boldsymbol{W} & -\boldsymbol{T}^{T} \\
\boldsymbol{T} & \mathbf{0}
\end{array}\right]^{-1}\left[\begin{array}{l}
\mathbf{0} \\
\boldsymbol{\tau}
\end{array}\right] .}
\end{aligned}
$$

Therefore, the control input command $\boldsymbol{u}$ can be simply calculated by using equation 25 and the azimuth angle, and the rotational speed of each propeller is calculated using equation 18 . However, this thrust allocation law should be changed in the case of a thruster failure or a thruster configuration change. If the azimuth thruster in the fore part was changed to a side tunnel thruster, which generated force in sway only, then equation 17 would change as shown in equation 25. The same thrust allocation algorithm from equation 18 through equation 25 can be applied. There is only one difference in applying equation 18 because the thrust of the tunnel thruster is not defined as the Euclidian norm.

$$
\boldsymbol{\tau}=\left[\begin{array}{lll}
X & Y & N
\end{array}\right]^{T}=\boldsymbol{T} \boldsymbol{f}=\boldsymbol{T} \boldsymbol{K} \boldsymbol{u},
$$

$$
\text { where, } \quad \begin{aligned}
\boldsymbol{T} & =\left[\begin{array}{ccc}
0 & 1 & 0 \\
1 & 0 & 1 \\
l_{x 1} & 0 & -l_{x 2}
\end{array}\right], \\
\boldsymbol{f} & =\left[\begin{array}{lll}
f_{1 y} & f_{2 x} & f_{2 y}
\end{array}\right]^{T}, \\
\boldsymbol{K} & =\left[\begin{array}{ccc}
a_{1}{ }^{2} & 0 & 0 \\
0 & a_{1}{ }^{2} & 0 \\
0 & 0 & a_{2}{ }^{2}
\end{array}\right], \\
\boldsymbol{u} & =\left[\begin{array}{lll}
u_{1 y} & u_{2 x} & u_{2 y}
\end{array}\right]^{T} .
\end{aligned}
$$


The term $\boldsymbol{f}^{T} \boldsymbol{W} \boldsymbol{f}$ in equation 20, which is the cost function for the thrust allocation in this study, is the square of the force and is often considered as power. Let's consider a negative damping force, where the force is a product of the negative damping coefficient and the velocity of the body as shown in equation 27.

$\boldsymbol{f}=\boldsymbol{A v}$,

where $\boldsymbol{A}$ is the negative damping coefficient matrix, which is assumed to be a diagonal matrix, $\boldsymbol{v}$ is the time derivative of the velocity vector $\boldsymbol{v}$ of the vessel. Thus, the term $\boldsymbol{f}^{T} \boldsymbol{W} \boldsymbol{f}$ in the cost function can be regarded as equivalent to the product of force and velocity, as shown in equation 28. $\boldsymbol{D}=\boldsymbol{W} \boldsymbol{A}$ is a constant and diagonal matrix and the vectors $\boldsymbol{f}$ and $\boldsymbol{v}$ are in parallel. This clearly shows that the square of forces $\boldsymbol{f}^{T} \boldsymbol{W} \boldsymbol{f}$ is proportional to the mechanical power $\boldsymbol{f} \cdot \boldsymbol{v}$. Therefore, the thrust allocation law in this study can be regarded as a minimum power consumption algorithm.

$\boldsymbol{f}^{T} \boldsymbol{W} \boldsymbol{f}=\boldsymbol{f}^{T} \boldsymbol{W} \boldsymbol{A} \boldsymbol{v}=\boldsymbol{f}^{T} \boldsymbol{D} \boldsymbol{v}=\boldsymbol{D} \boldsymbol{f} \cdot \boldsymbol{v}$.

For the thrust allocation of the main propeller with the rudder, an iterative thrust allocation algorithm is developed. In the previous discussion of thrust allocation, two azimuth thrusters were considered. There was no constraint on the azimuth angle. However, the constraint on the azimuth angle does exist in the main propeller with the rudder operation, as illustrated in Figure 3. The simplest way of considering this propeller in the thrust allocation is to regard it as two large tunnel thrusters which generate forces in the surge and sway directions, respectively. However, in reality, this is not the case. Notably, the forces in the surge and sway directions generated by the main propeller with the rudder have strong coupling effects from the rudder angle. This means that if the surge force is increased by the main propeller with a significant rudder angle, then an undesirable sway force is also increased naturally, and vice versa. Moreover, this strong coupling effect sometimes causes unstable performance of the DP system. In this study, an iterative thrust allocation algorithm is used to deal with the highly coupled nonlinear characteristics of the main propeller with the rudder. The main idea of this algorithm is that the propeller and rudder are treated as one azimuth thruster with constraints on the azimuth angle as shown in Figure 3. The algorithm followed the procedure proposed below:

- Find the optimal thrust allocation solution considering the main propeller with the rudder as one virtual azimuth thruster.

- Check whether the azimuth angle of the virtual azimuth thruster exceeds the limitation on the rudder operation.

- If the azimuth angle of the virtual thruster exceeds the limitation, then set the azimuth angle of the virtual thruster to the closest limitation.

- Calculate the component forces and moment of the virtual azimuth thruster with the closest azimuth angle limitation.

- Extract the effects of the calculated component forces and moment of the virtual thruster from equation 19 to create a reduced order problem.

- Find a new optimal solution for the thrust allocation with the reduced order problem. 


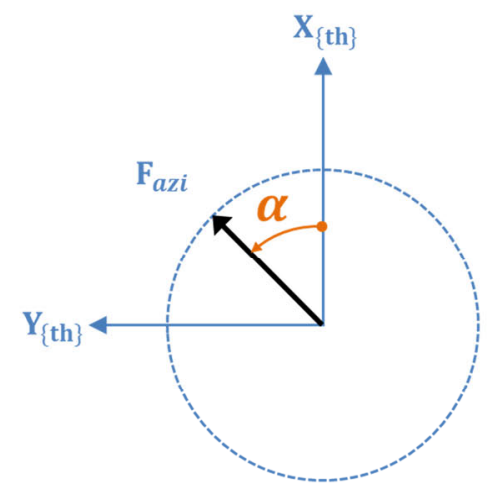

Azimuth Thruster

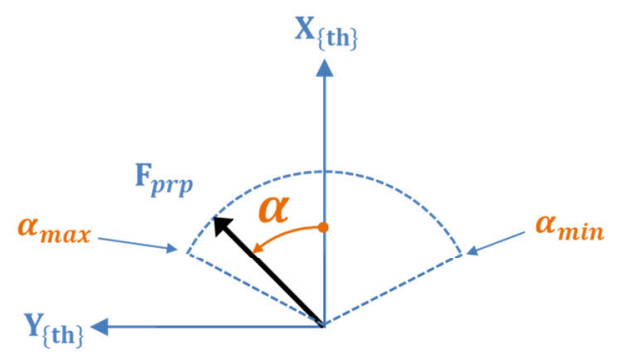

Main Propeller with Rudder

Fig. 3 Local coordinates of azimuth thruster and main propeller with rudder

\section{Model test and numerical simulation}

A set of experiments was conducted in the ocean engineering basin (OEB) of KRISO to estimate the station-keeping performance of a DP shuttle tanker with the PD and anti-windup I controllers and the thrust allocation algorithm, including the rudder considerations introduced in the previous section of this paper. The main particulars of the DP shuttle tanker are shown in Table 1. The DP shuttle tanker had six thruster systems: two side tunnel thrusters and one azimuth thruster at the fore, and one azimuth thruster, one side tunnel thruster, and one main propeller with a rudder at the aft of the vessel, as shown in Figure 4.

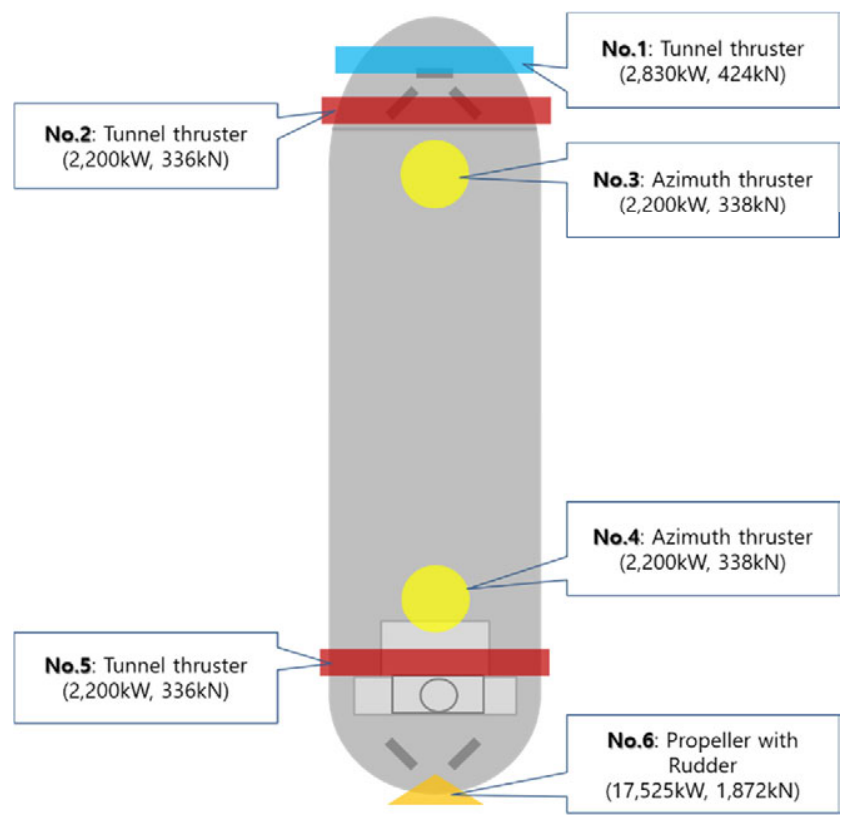

Fig. 4 Layout of thruster systems of the dynamic positioning shuttle tanker

The environmental conditions were selected after the consideration of the PETROBRAS requirements for DP vessels operating in the Brazilian waters. Three environmental conditions were selected in this study: 
Env-I: No current-off condition (collinear condition),

$$
\begin{aligned}
& H_{s}=0.5 \mathrm{~m}, T_{p}=8.64 \mathrm{~s}, \operatorname{JONSWAP}(\gamma=1), \beta_{\text {wave }}=180^{\circ}, \\
& V_{w}=21.0 \mathrm{~m} / \mathrm{s}, \beta_{w}=180^{\circ} \\
& V_{c}=1.1 \mathrm{~m} / \mathrm{s}, \beta_{c}=180^{\circ}
\end{aligned}
$$

Env-II: $20^{\circ}$ current-off condition,

$$
\begin{aligned}
& H_{s}=0.5 \mathrm{~m}, T_{p}=8.64 \mathrm{~s}, \operatorname{JONSWAP}(\gamma=1), \beta_{\text {wave }}=180^{\circ}, \\
& V_{w}=21.0 \mathrm{~m} / \mathrm{s}, \beta_{w}=180^{\circ} \\
& V_{c}=1.1 \mathrm{~m} / \mathrm{s}, \beta_{c}=160^{\circ}
\end{aligned}
$$

O Env-III: $35^{\circ}$ current-off condition,

$$
\begin{aligned}
& H_{s}=0.5 \mathrm{~m}, T_{p}=8.64 \mathrm{~s}, \operatorname{JONSWAP}(\gamma=1), \beta_{\text {wave }}=180^{\circ}, \\
& V_{w}=21.0 \mathrm{~m} / \mathrm{s}, \beta_{w}=180^{\circ}, \\
& V_{c}=1.1 \mathrm{~m} / \mathrm{s}, \beta_{c}=145^{\circ}
\end{aligned}
$$

where $H_{s}, T_{p}$ are the significant wave height and peak period of the wave. In the simulation and experiment of the DP analysis in this study, long crested waves are used and the wave spectrum for the collinear current condition is given in Figure 5. The JONSWAP wave spectrum with the peakedness parameter $\gamma$ value 1 is used in the experiment and numerical analysis.

Table 1 Main particulars of the dynamic positioning shuttle tanker

\begin{tabular}{l|c|c}
\hline \multicolumn{1}{c|}{ Item } & Unit & Value \\
\hline Length overall $(\boldsymbol{L})$ & $\mathrm{m}$ & 278.0 \\
\hline Breadth $(\boldsymbol{B})$ & $\mathrm{m}$ & 48.7 \\
\hline Draft in full load condition $(\boldsymbol{T})$ & $\mathrm{m}$ & 17.0 \\
\hline Displacement in full load condition & $\mathrm{m}^{3}$ & $1.78 \times 10^{5}$ \\
\hline Block coefficient $\boldsymbol{C}_{\boldsymbol{b}}$ & - & 0.77 \\
\hline
\end{tabular}

KRISO developed three DP thruster systems for the model test: the azimuth thruster, the tunnel thruster, and the main propeller with the rudder. An azimuth thruster system is shown in Figure 5; it consists of the mechanical thruster and its electrical controller. All thruster systems communicate with the DP controller using the RS-232 protocol or the TCP/IP protocol. 


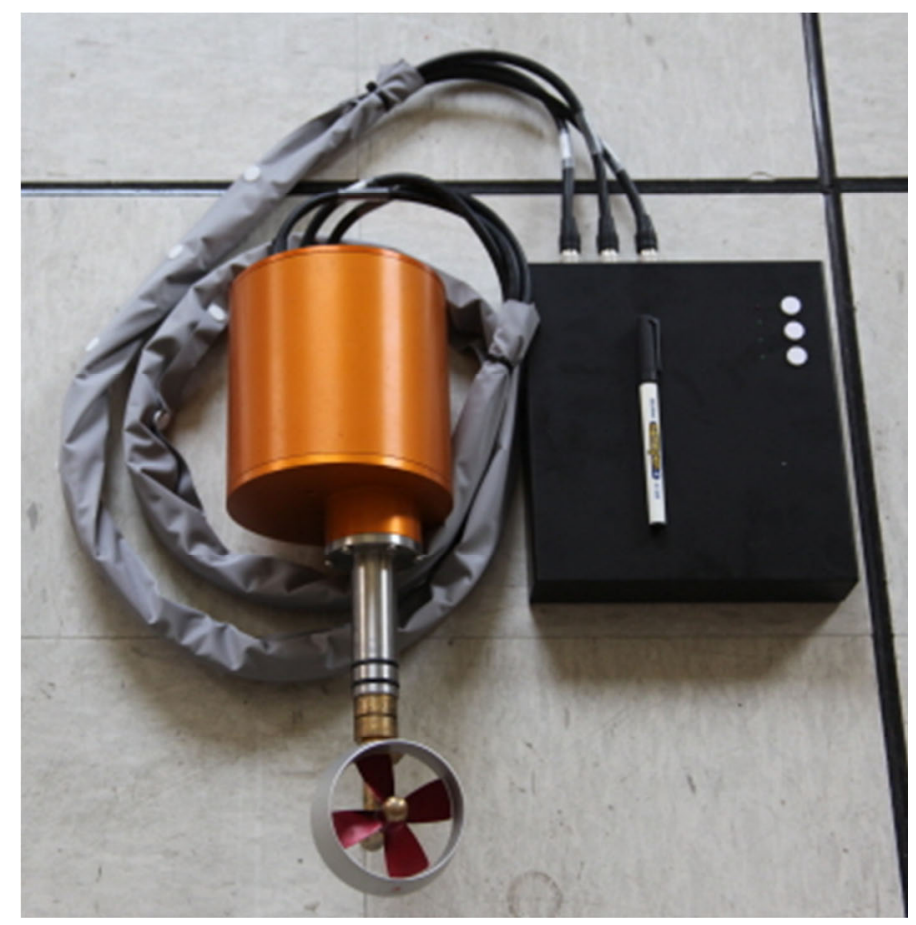

Fig. 5 An azimuth thruster system: thruster and its controller

The model test for the evaluation of the station-keeping performance of a DP vessel consists of several individual experiments. The procedure for the DP model test is shown below:

- Model fabrication;

- Ocean environment calibration;

- Bollard pull test;

- Wind load adjustment;

- Gain tuning test;

- Main test, including the worst single failure case.

In the model fabrication, a model of the vessel was made of wood and fibre-reinforced plastic and other materials. The thruster systems, power distribution system, and other equipment used to run the DP system were installed inside the vessel model. Before conducting the DP model test, all ocean environmental conditions, i.e. the wind, waves, and current conditions, were calibrated to have the designated characteristics. The bollard pull test was conducted to find out the characteristics of the thruster system. The wind field generated in the DP model test was not the same as the real wind field in the ocean. Thus, the wind load acting on the DP vessel was adjusted by adding or extracting a superstructure model of the DP vessel model or by changing the wind speed. The DP control gain tuning procedure was carried out in calm water conditions to have designated station-keeping performance. Then, the main DP model tests were conducted in various environmental conditions, and under several failure cases of the DP system, such as a no failure case, thruster failure cases, and the worst single failure case. The DP shuttle tanker model during the main DP model tests is shown in Figure 6. 


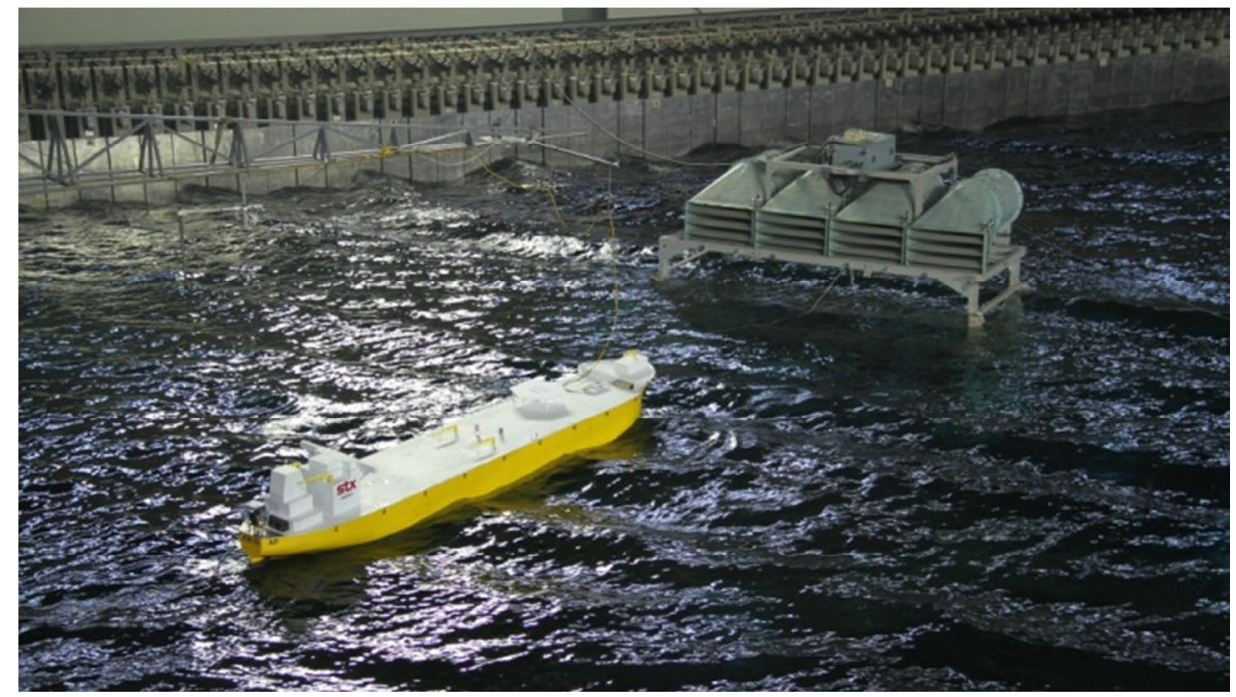

Fig. 6 The dynamic positioning shuttle tanker model during the model test in the ocean engineering basin of KRISO

\section{Results and discussion}

A set of experiments and numerical analyses for the evaluation of the station-keeping performance of a DP shuttle tanker was conducted in KRISO's OEB. A PD controller with an anti-windup I control algorithm and a thrust allocation method, including considerations for the main propeller with the rudder, were utilized in both the experiments and the numerical simulations. A fairly good overall agreement was observed between the results of the experiment and the numerical analysis, with the same PD gain setting for the DP control system as listed in Table 2. It should be noted that the I gain $\boldsymbol{K}_{I}$ and the corresponding time window size $T_{I}$ slightly differed in the model test and the simulation. The I gain settings for the simulation are intentionally selected to have similar steady-state errors in the surge direction under Env-I (no current-off condition) as shown in Table 3. This intentional gain tuning of I controller is for direct comparison of the motion responses of the DP vessel in the experiment and simulation results. It is reasonable to have a minimum basis of condition for comparison. The footprints of the vessel motion under various failure conditions of a DP system were acquired in experiments and numerical simulations, as shown in Figure 7 through Figure 9; they were used for the failure mode effect analysis (FMEA). When all thrusters were working properly under the Env-I condition, in which all environmental forces were acting in the same direction, the DP shuttle tanker maintained the position of the bow loading system (BLS) within a predefined watch circle, with minor motion as shown in Figure 7. Note that the radius of the BLS motion circle was about $13.1 \mathrm{~m}$ in the experiment and $6.8 \mathrm{~m}$ in the numerical simulation, which are both reasonably small radii compared to the radius of the predefined watch circle, i.e. $50 \mathrm{~m}$. The worst single failure case from the FMEA, which is the failure case of No.1 bow tunnel thruster and No. 3 bow azimuth thruster, did not make the DP shuttle tanker drift-off under the Env-I condition, and even showed similar stationkeeping performance to the no thruster failure case as shown in Figure 8. However, when the main propeller system was out of order, the DP shuttle tanker showed the drift-off response in the experiment, and a significantly big motion response in the simulation, as shown in Figure 9. The radii of the BLS motion circles were observed to be $37.0 \mathrm{~m}$ in the experiment and $32.7 \mathrm{~m}$ in the simulation.

It is noticeable that quite different sway and yaw motions are observed in the experiment and simulation results. Significant sway and yaw motions are observed in the experimental result; on the other hand, no sway and yaw motions are shown in the simulation 
result. This significant differences between the model test and the simulation are caused by several factors. Firstly, uncertainty in the modelling of the environmental forces created by wind, wave, and current is the main cause of the differences. In the simulation, the uniform environmental field is considered, such as the same wind and current speed and direction are assumed and modelled. However, in the model test, which is closer to the real sea condition than the simulation, such uniform environmental field could not be realized in the space and time domain. Only the mean value concept can represent the uniformness of environmental field. Therefore, in the experiment, even in the collinear environment condition of Env-I, excitation forces in the sway and yaw directions possibly exist and these caused the significant motion in these directions. Secondly, uncertainty in the modelling of the thruster system is the other cause of the differences. In the simulation, a simple $1^{\text {st }}$ order thruster system model is used. This $1^{\text {st }}$ order model could not fully represent the thruster system used in the experiment. The transient status of the thruster in between serial commands in particular can be quite different. When an azimuth thruster is commanded to change its azimuth angle from $90^{\circ}$ to $-90^{\circ}$ with a certain propeller rotational speed, the thruster produces forces in all directions during the azimuth angle change; and this can cause the excitation in the sway and yaw directions.

Table 2 Main particulars of the dynamic positioning shuttle tanker

\begin{tabular}{cc|c|c|c|c}
\hline Item & & $\begin{array}{c}\boldsymbol{K}_{\boldsymbol{P}} \\
{[\mathrm{kN} / \mathrm{m}]} \\
{[\mathrm{kN} \cdot \mathrm{m} / \mathrm{rad}]}\end{array}$ & $\begin{array}{c}\boldsymbol{K}_{\boldsymbol{D}} \\
{[\mathrm{kN} \cdot \mathrm{s} / \mathrm{m}]} \\
{[\mathrm{kN} \cdot \mathrm{m} \cdot \mathrm{s} / \mathrm{rad}]}\end{array}$ & $\begin{array}{c}\boldsymbol{K}_{\boldsymbol{I}} \\
{[\mathrm{kN} / \mathrm{m}]} \\
{[\mathrm{kN} \cdot \mathrm{m} / \mathrm{m}]}\end{array}$ & $\begin{array}{c}\boldsymbol{T}_{\boldsymbol{I}} \\
{[\mathrm{s}]}\end{array}$ \\
\hline \multirow{3}{*}{ Model test } & Surge & $5.1 \times 10^{1}$ & $4.4 \times 10^{3}$ & $8.0 \times 10^{0}$ & 71 \\
\cline { 2 - 6 } & Sway & $7.6 \times 10^{1}$ & $6.6 \times 10^{3}$ & $8.0 \times 10^{0}$ & 71 \\
\cline { 2 - 6 } & Yaw & $3.5 \times 10^{5}$ & $3.1 \times 10^{6}$ & $2.0 \times 10^{0}$ & 71 \\
\hline \multirow{3}{*}{ Simulation } & Surge & $5.1 \times 10^{1}$ & $4.4 \times 10^{3}$ & $5.1 \times 10^{-1}$ & 100 \\
\cline { 2 - 6 } & Sway & $7.6 \times 10^{1}$ & $6.6 \times 10^{3}$ & $7.6 \times 10^{-1}$ & 100 \\
\cline { 2 - 6 } & Yaw & $3.5 \times 10^{5}$ & $3.1 \times 10^{6}$ & $3.6 \times 10^{3}$ & 100 \\
\hline
\end{tabular}


YS Kim, JP Hong, YH Kim

HG Sung, J. Kim
Design and Experimental Validation of a Control System for Dynamic Positioning of a Shuttle Tanker
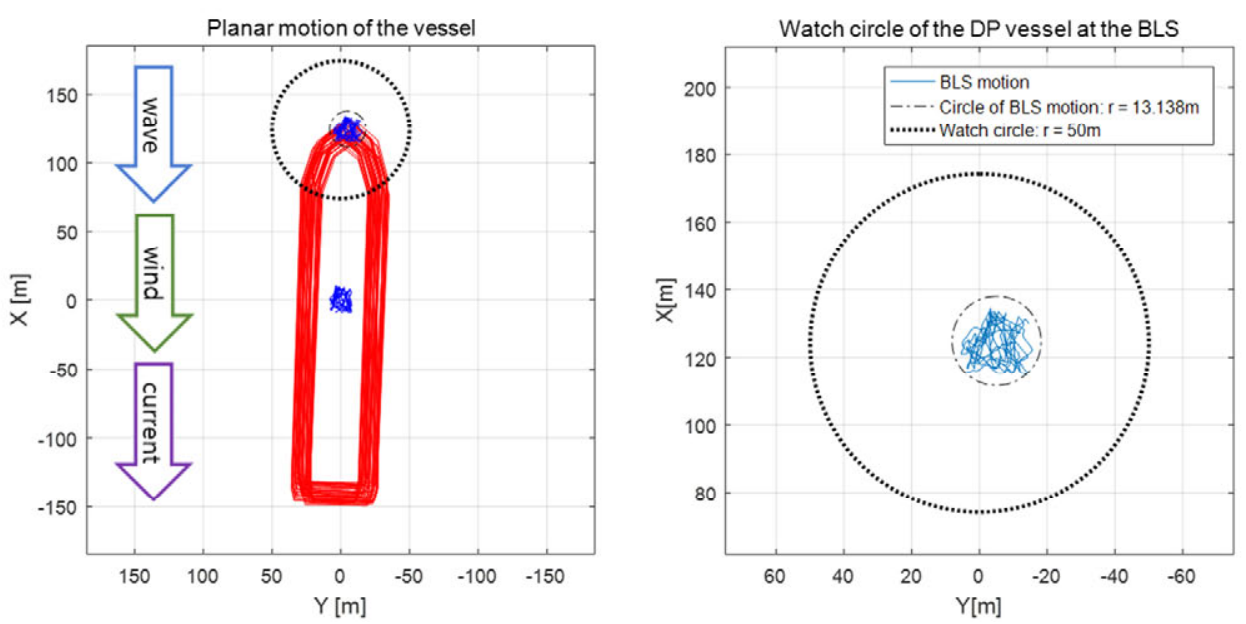

(a) Model test
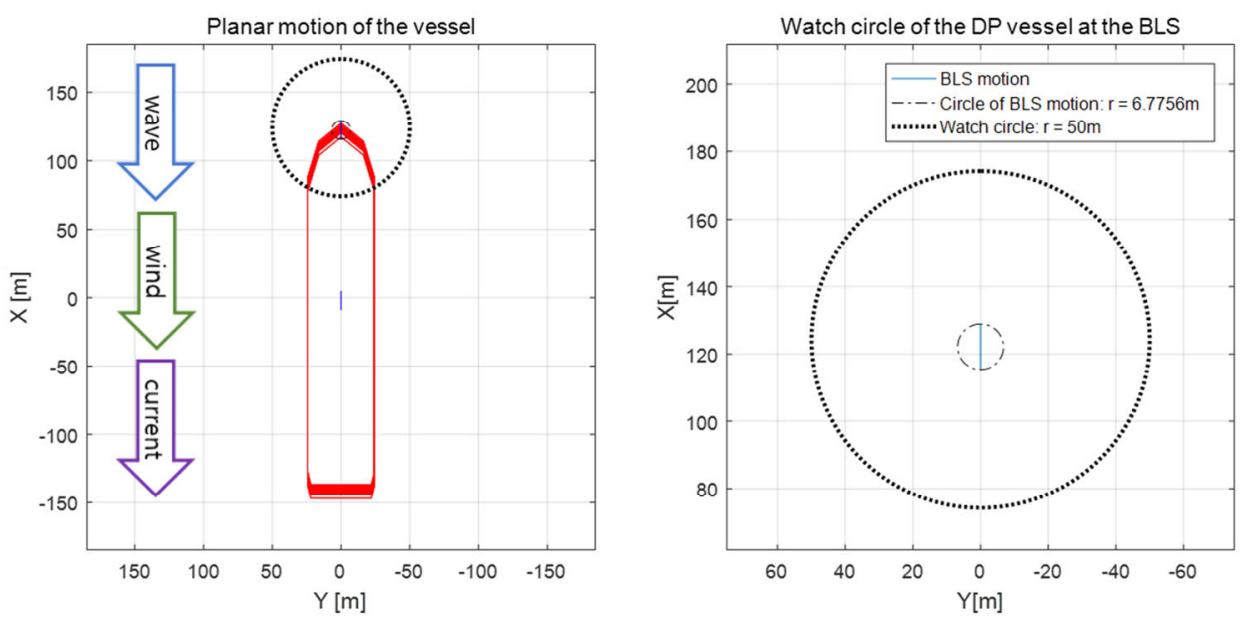

(b) Numerical simulation

Fig. 7 Footprints of the vessel motion and trajectory of the bow loading system (BLS): Env-I / no thruster failure 

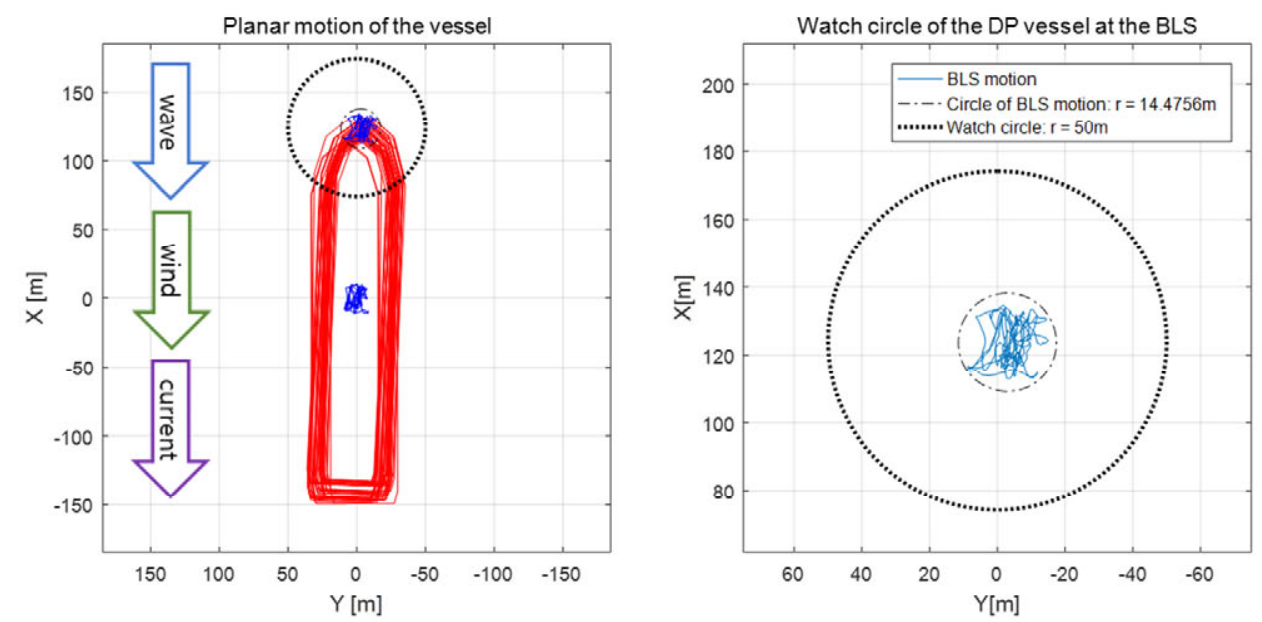

(a) Model test
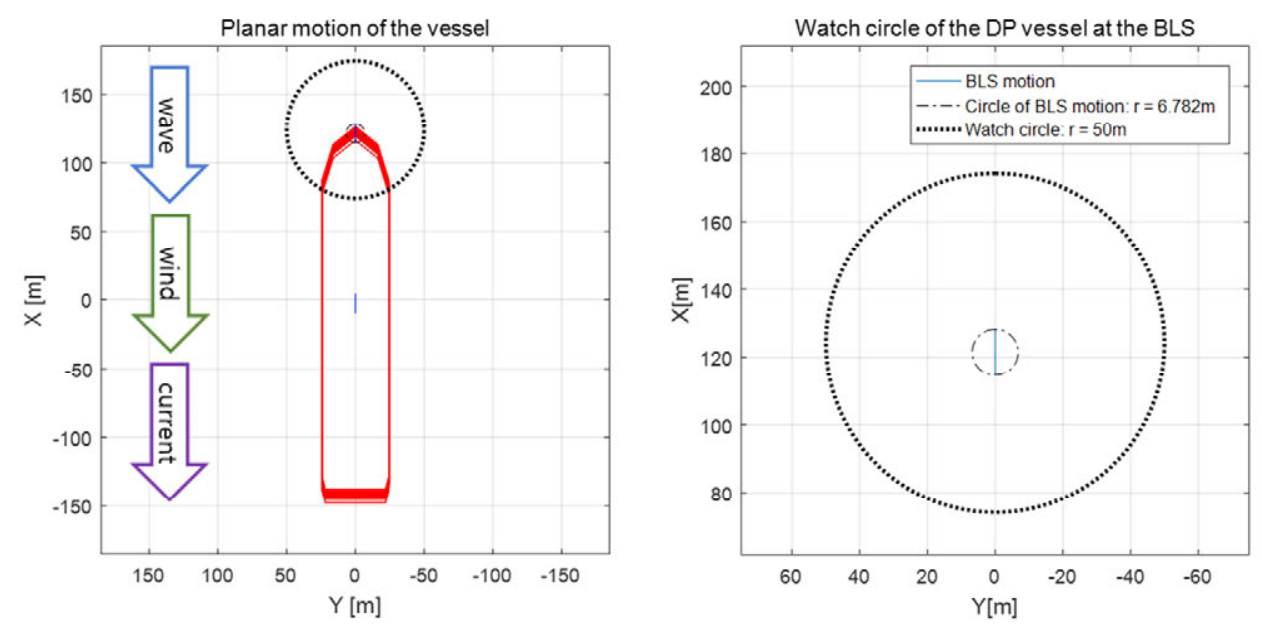

(b) Numerical simulation

Fig. 8 Footprints of the vessel motion and trajectory of the bow loading system (BLS): Env-I / the worst single failure (No.1 bow tunnel thruster and No. 3 bow azimuth thruster failure) 
YS Kim, JP Hong, YH Kim

HG Sung, J. Kim
Design and Experimental Validation of a Control System for Dynamic Positioning of a Shuttle Tanker
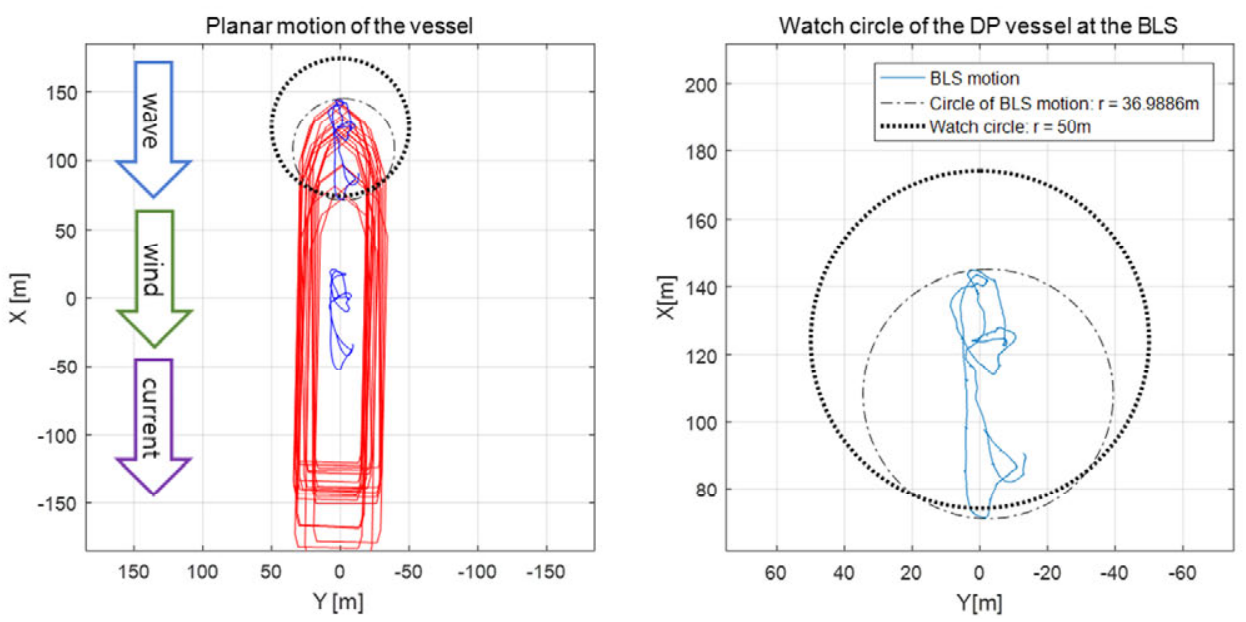

(a) Model test
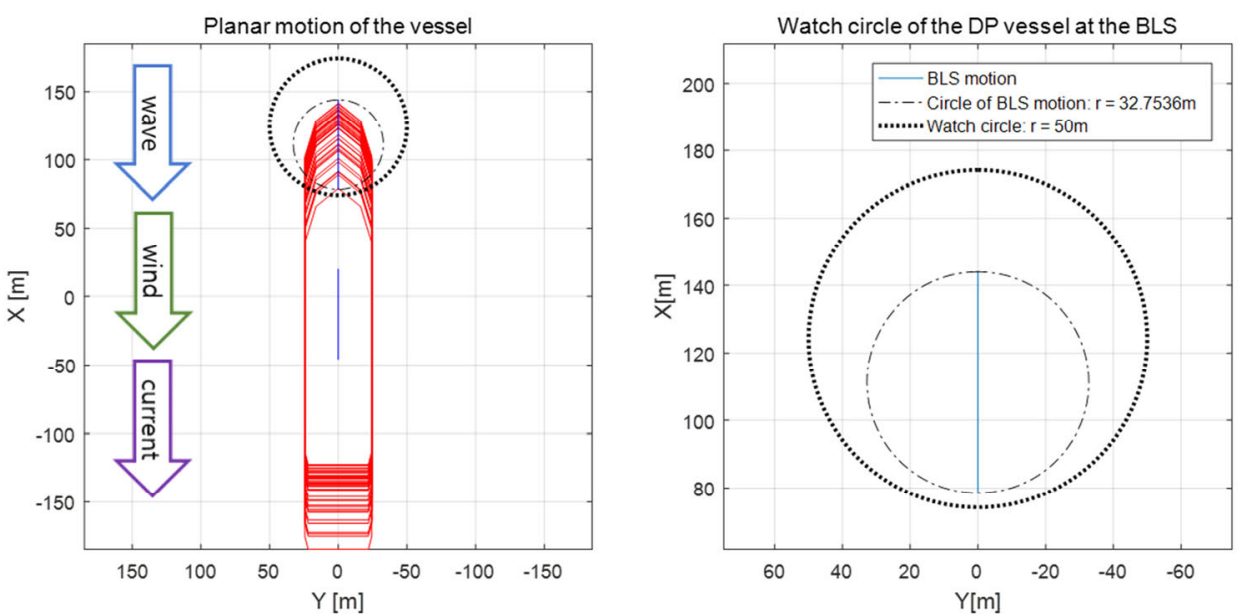

(b) Numerical simulation

Fig. 9 Footprints of the vessel motion and trajectory of the bow loading system (BLS): Env-I / No. 6 main propeller with a rudder failure

The station-keeping performance of the DP shuttle tanker under the current-off conditions was examined. Two current-off conditions were considered: the $20^{\circ}$ current-off condition (Env-II) and the $35^{\circ}$ current-off condition (Env-III). When all thrusters were working under the Env-II condition, the DP shuttle tanker was stable and showed good station-keeping performance in both the model test and the simulation, as shown in Figure 10. The radii of the circles of the BLS motion were observed at $12.8 \mathrm{~m}$ in the model test and at $7.0 \mathrm{~m}$ in the simulation. On the other hand, under the Env-III condition, the DP shuttle tanker showed fairly stable station-keeping performance with all thrusters working, as shown in Figure 11. The radii of the circle of the BLS motion differed significantly in the experiment and in the simulation. The radius of $33.5 \mathrm{~m}$ was observed in the model test, while only that of $7.3 \mathrm{~m}$ was observed in the simulation. This significant difference between the model test result and the simulation result was mainly caused by the effect of the $1^{\text {st }}$ order wave force, which was not considered in the simulation but was naturally considered in the model test. The dynamics of the thrusters also had a role in causing this difference. In the simulation, a simple $1^{\text {st }}$ order model for thrusters was utilized without any consideration for the thruster interactions. However, in the model test, the dynamics of the thruster system has apparent effects at a certain level; only the power system dynamics was not considered in the model test. 
The distance of the BLS from the centre of the watch circle and the planar motion of the vessel are shown in Figure 12. A maximum distance of $45.4 \mathrm{~m}$ and a mean distance of $15.2 \mathrm{~m}$ were observed in the experiment, while a maximum distance of $10.27 \mathrm{~m}$ and a mean distance of $2.22 \mathrm{~m}$ were observed in the simulation. A sudden change in the BLS distance was observed in the experiment when a big wave hit the vessel. On the other hand, no such rapid BLS distance change was observed in the numerical simulation because the slow-drift $2^{\text {nd }}$ order wave force was the only wave force considered in the numerical simulation. Although this is the limitation of the numerical simulation for evaluating the station-keeping performance of the DP system, the results of the time-domain simulation show the overall behaviour of the DP system, as shown in the above comparison between the experimental results and the numerical simulation results.
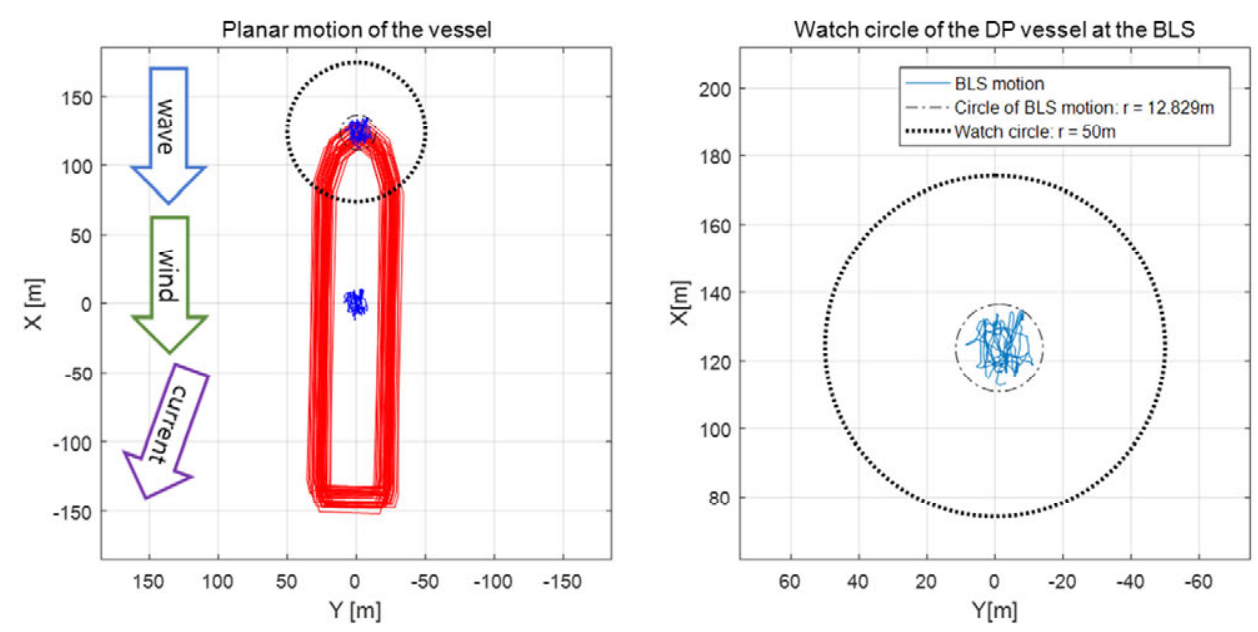

(a) Model test
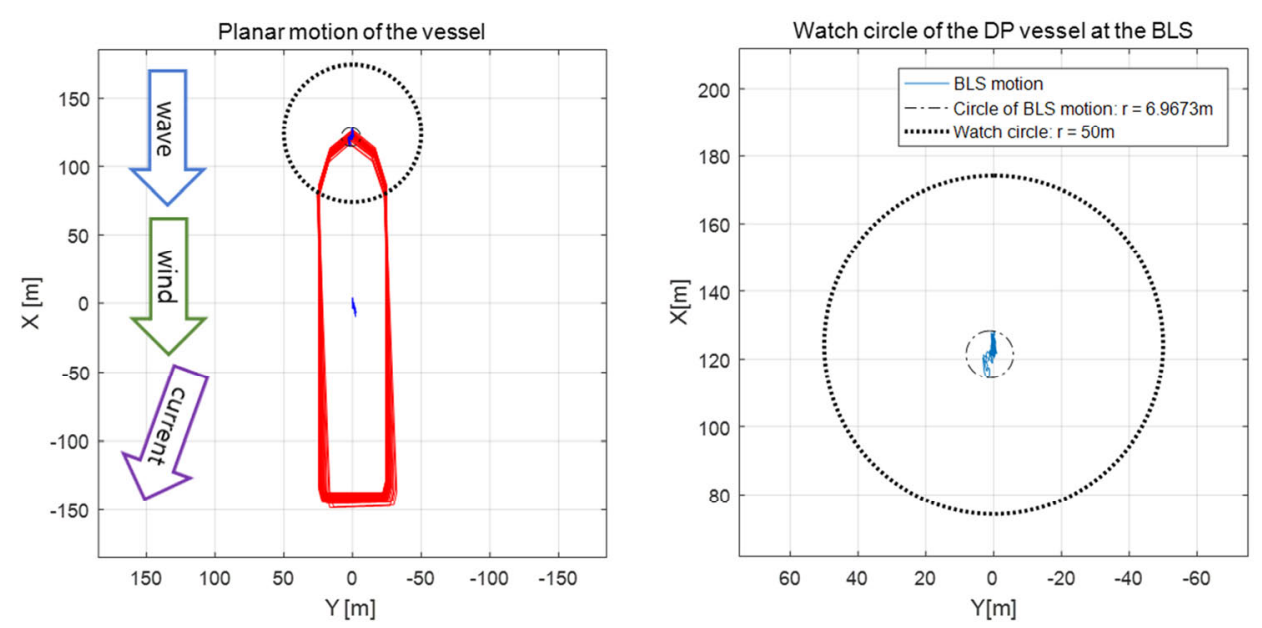

(b) Numerical simulation

Fig. 10 Footprints of the vessel motion and trajectory of the bow loading system (BLS): Env-II (20 current-off condition), no thruster failure 

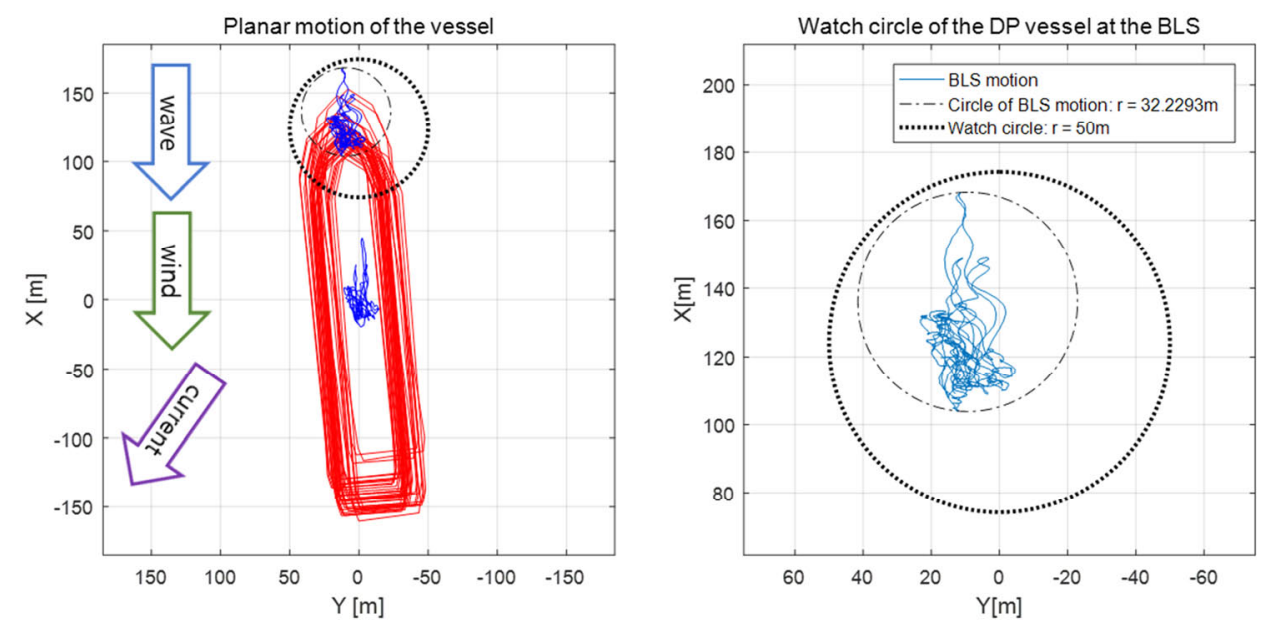

(a) Model test
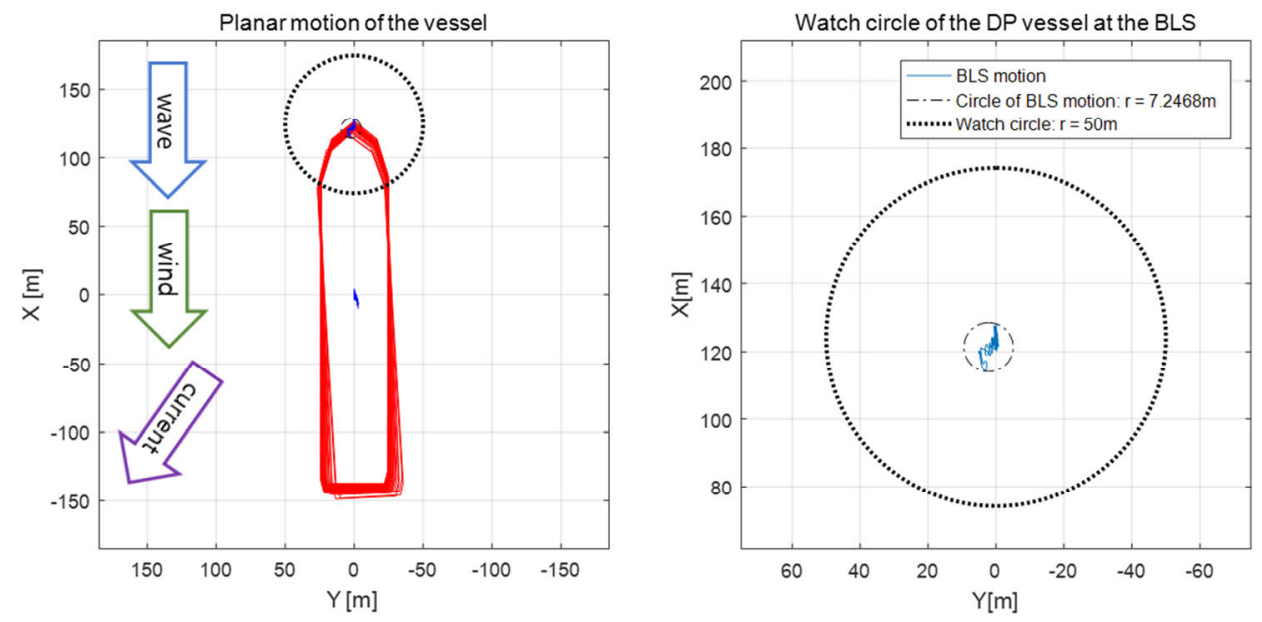

(b) Numerical simulation

Fig. 11 Footprints of the vessel motion and trajectory of the bow loading system (BLS): Env-III ( $35^{\circ}$ current-off condition), no thruster failure

The statistical results of the vessel motion are given in Tables 3 and 4. The mean values of the surge and sway motions in the model test and in the numerical simulation were similar. On the other hand, the mean values of the yaw motion in the experiment were slightly greater than those in the simulation. It is thought that the $1^{\text {st }}$ order wave force and the thruster dynamics in the experiment caused these yaw motion differences. Though these were small differences, their effects on the distance from the BLS to the centre of the watch circle were significant, as shown in Figure 12. This implies that the yaw motion control in the DP shuttle tanker operation was very important. The standard deviation (STD) values of all the motions and distances in the experiment were slightly greater than those in the simulation. This implies that greater oscillatory motions take place in the experiment, and that the $1^{\text {st }}$ order wave force, which did not exist in the numerical simulation, caused these oscillations. Especially, the STD value of the surge motion in the $35^{\circ}$ current-off condition was much bigger than the one in the $35^{\circ}$ current-off condition because larger wave forces acted on the vessel. So it can be stated that the consideration of the $1^{\text {st }}$ order wave force should be required in the numerical simulation for having closer similitude with real situations in DP operation. 
Table 3 Mean values of the vessel motion

\begin{tabular}{cc|r|r|r|r}
\hline \multicolumn{2}{c|}{ Condition } & \multicolumn{1}{c|}{$\begin{array}{c}\text { Surge } \\
{[\mathrm{m}]}\end{array}$} & \multicolumn{1}{c|}{$\begin{array}{c}\text { Sway } \\
{[\mathrm{m}]}\end{array}$} & \multicolumn{1}{c|}{$\begin{array}{c}\text { Yaw } \\
{\left[{ }^{\circ}\right]}\end{array}$} & \multicolumn{1}{c}{$\begin{array}{c}\text { Distance } \\
{[\mathrm{m}]}\end{array}$} \\
\hline \multirow{3}{*}{ Model test } & No current-off & -0.42 & 0.02 & -2.08 & 7.43 \\
\cline { 2 - 6 } & $20^{\circ}$ current-off & -0.14 & 0.17 & -0.97 & 6.24 \\
\cline { 2 - 6 } & $35^{\circ}$ current-off & -0.78 & -0.07 & -0.97 & 15.20 \\
\hline \multirow{3}{*}{ Simulation } & No current-off & -0.42 & 0.00 & 0.00 & 1.75 \\
\cline { 2 - 6 } & $20^{\circ}$ current-off & -1.13 & -0.46 & 0.36 & 2.11 \\
\cline { 2 - 6 } & $35^{\circ}$ current-off & -1.13 & -0.71 & 0.57 & 2.22 \\
\hline
\end{tabular}

Table 4 Standard deviations of the vessel motion

\begin{tabular}{rl|r|r|r|r}
\hline \multicolumn{2}{c|}{ Condition } & \multicolumn{1}{c|}{$\begin{array}{c}\text { Surge } \\
{[\mathrm{m}]}\end{array}$} & $\begin{array}{c}\text { Sway } \\
{[\mathrm{m}]}\end{array}$ & \multicolumn{1}{c|}{$\begin{array}{c}\text { Yaw } \\
{\left[{ }^{\circ}\right]}\end{array}$} & \multicolumn{1}{c}{$\begin{array}{c}\text { Distance } \\
{[\mathrm{m}]}\end{array}$} \\
\hline \multirow{3}{*}{ Model test } & No current-off & 4.90 & 4.24 & 0.83 & 3.53 \\
\cline { 2 - 6 } & $20^{\circ}$ current-off & 5.11 & 3.68 & 0.49 & 2.50 \\
\cline { 2 - 6 } & $35^{\circ}$ current-off & 11.00 & 5.53 & 1.05 & 6.22 \\
\hline \multirow{3}{*}{ Simulation } & No current-off & 2.26 & 0.00 & 0.00 & 1.49 \\
\cline { 2 - 6 } & $20^{\circ}$ current-off & 2.27 & 0.58 & 0.54 & 1.63 \\
\cline { 2 - 6 } & $35^{\circ}$ current-off & 2.27 & 0.77 & 0.85 & 1.81 \\
\hline
\end{tabular}
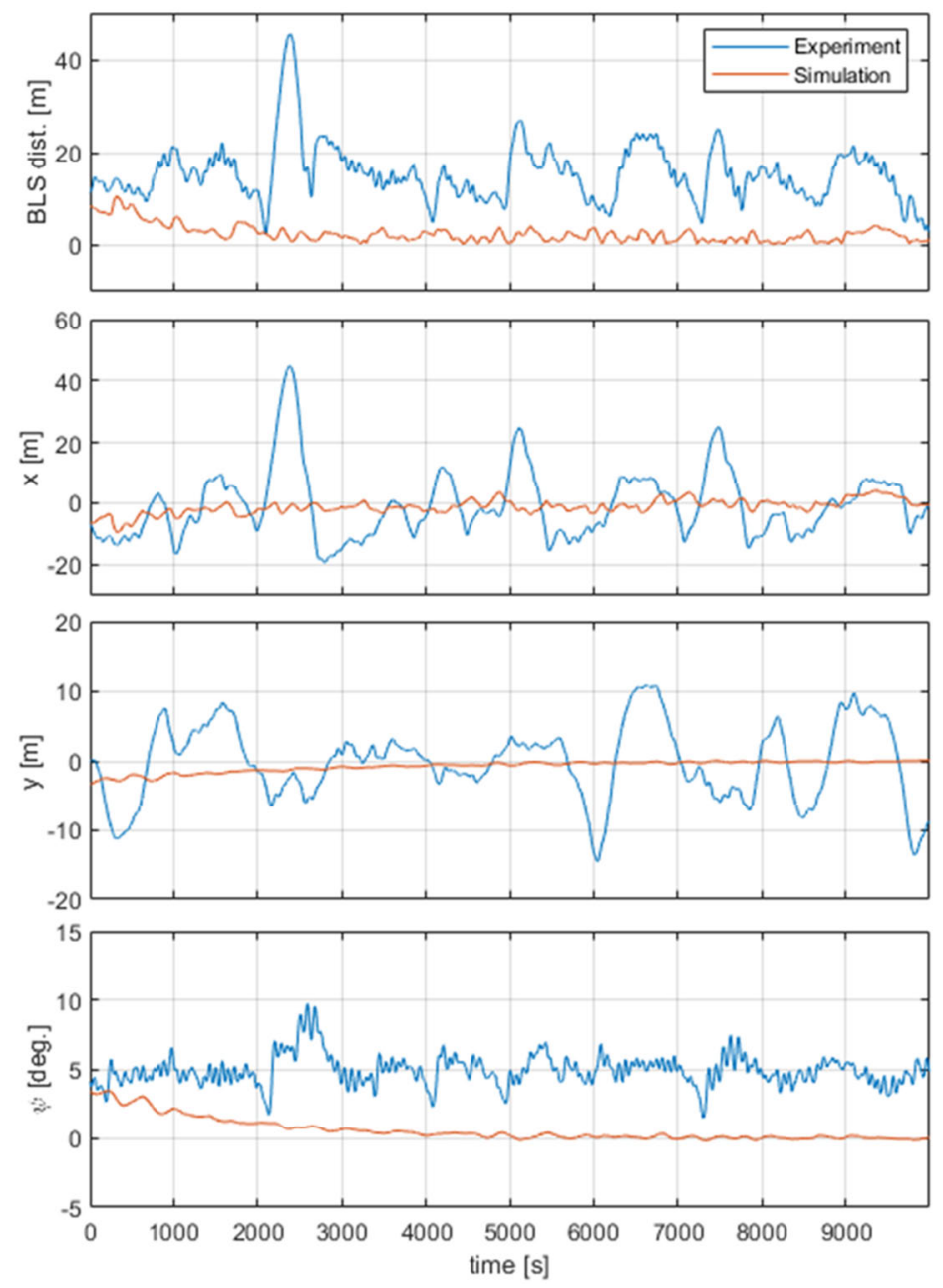

Figure 12 Distance of the BLS from the centre of the watch circle, and planar motion of the vessel: Env-III ( $35^{\circ}$ current-off condition), no thruster failure 


\section{Conclusions}

A method for the evaluation of dynamic positioning (DP) performance for a shuttle tanker has been discussed in this paper through experimentation and numerical analysis. This paper has presented a methodology for the model testing and numerical analysis for the station-keeping performance evaluation of a DP shuttle tanker. A review of the numerical model for the DP simulation has been given. A new anti-windup I control algorithm for the suppression of the steady-state error has been introduced, and a thrust allocation algorithm, including the treatment of the main propeller with a rudder, has been developed. From the experimental and numerical simulation results, the DP control algorithm, including the thrust allocation method, has been validated. Reasonable agreement between the experimental results and the time-domain simulation results is provided in this paper.

\section{Acknowledgment}

This study was supported by both a grant from the National R\&D Project 'Development of an electric-powered car ferry and a roll-on/roll-off power supply system' funded by the Ministry of Oceans and Fisheries, Korea (PMS4700) and the National R\&D Project 'Establishment of Research Infrastructure for Deepsea Offshore Engineering Basin and Development of Offshore Structure PreFEED technologies' funded by the Ministry of Oceans and Fisheries, Korea (PMS3850). The authors would especially like to thank the KRISO ocean engineering basin technicians for their dedicated efforts.

\section{REFERENCES}

[1] Sørensen AJ. 2011. A survey of dynamic positioning control systems. Annual reviews in control. 35(1):123-136. https://doi.org/10.1016/j.arcontrol.2011.03.008

[2] Tannuri EA, Morishita HM. 2006. Experimental and numerical evaluation of a typical dynamic positioning system. Applied Ocean Research. 28(2): 133-146. https://doi.org/10.1016/j.apor.2006.05.005

[3] Fossen TI. 1994. Guidance and control of ocean vehicles. United Kingdom: JOHN WILEY \& SONS; ISBN-13: 978-0471941132.

[4] Fossen TI. 2011. Handbook of marine craft hydrodynamics and motion control. United Kingdom: JOHN WILEY \& SONS; ISBN-13: 978-1119575054. https://doi.org/10.1002/9781119994138

[5] Tannuri EA, Agostinho AC, Morishita HM, Moratelli Jr L. 2010. Dynamic positioning systems: An experimental analysis of sliding mode control. Control Engineering Practice. 18(10):1121-1132. https://doi.org/10.1016/j.conengprac.2010.06.007

[6] El-Hawary F. 2000. The ocean engineering handbook. United Kingdom: CRC Press; ISBN-13: 9780367397692. https://doi.org/10.1201/9781420036732

[7] Sørensen AJ. 2012. Marine control systems propulsion and motion control of ships and ocean structures lecture notes. Norway: NTNU. No.: UK-13-76.

[8] Kim YS, Kim YH, Sung HG, Kim J. 2013. A robust dynamic positioning control algorithm and its validation through experiment and numerical simulation. In: ISOPE, editor. Proceedings of $23^{\text {rd }}$ International Offshore and Polar Engineers, ISOPE; Jun 30 - Jul 5; Alaska, USA, p. 979-986.

[9] Choi YR, Hong SY, Choi HS. 2001. An analysis of second-order wave forces on floating bodies by using a higher-order boundary element method, Ocean Engineering. 28(1): 117-138. https://doi.org/10.1016/ S0029-8018(99)00064-5

[10] SNAME. 1950. Nomenclature for treating the motion of a submerged body through a fluid. USA: The Society of Naval Architects and Marine Engineers. No.: Technical and Research Bulletin No.1-5.

[11] Faltinsen OM. 1993. Sea loads on ships and offshore structures. United Kingdom: Cambridge University Press; ISBN-13: 978-0521458702.

[12] OCIMF. 1994. Prediction of wind and current loads on VLCCs. United Kingdom: Witherby Publishing Group; ISBN-13: 978-1856090421.

[13] Artyszuk J. 2004. A uniform current in ship manoeuvring mathematical model. Annual of Navigation. 8: 67-76. 
[14] Skejic R, Breivik M, Berg TE. 2011. Investigating ship maneuvers around a floating structure under the influence of a uniform current in deep and calm water, In: Second International Conference on Ship Manoeuvring in Shallow and Confined Water: Ship to Ship Interaction, editor. Pettersen B, Beng TE, Eloot K, Vantorre M; May 18-20; London, UK, p. 339-350.

[15] Newman JN. 1974. Second-order, slowly-varying forces on vessels in irregular waves, In: International Symposium of Dynamics of Marine Vehicles and Structures in Waves. USA: MIT. No.: MIT Paper 19.

Submitted: $\quad 22.3 .2021$

Accepted: $\quad 05.7 .2021$
Young-Shik Kim

Jang-Pyo Hong

Yun-Ho Kim*

Hong-Gun Sung

Korea Research Institute of Ships and

Ocean Engineering, Daejeon, South Korea Jinwhan Kim

KAIST, Daejeon, South Korea

*Corresponding author:

yunhokim@kriso.re.kr 